\title{
Analysis of Polarization Properties of Shallow Metallic Gratings by an Extended Rayleigh-Fano Theory
}

\author{
Masato Koike \\ Center for X-Ray Optics \\ Accelerator and Fusion Research Division \\ Lawrence Berkeley Laboratory \\ University of California \\ Berkeley, California 94720
}

Takeshi Namioka

Research Institute for Scientific Measurements

Tohoku University

Sendai 980

Japan

June 1991

This repor has been reproduced dirently from the best available copy.

This work was supported by the Director, Office of Energy Research, Office of Basic Energy Sciences, Materials Sciences Division, of the U.S. Department of Energy under Contract No. DE-AC03-76SF00098. 
Analysis of polarization properties of shallow metallic gratings by an extended Rayleigh-Fano theory

\author{
Masato Koike
}

Center for X-Ray Optics, Lawrence Berkeley Laboratory

University of California, Berkeley, California 94720

Takeshi Namioka

Resenrch Institute for Scientific Mensurements

Tohoku University, Sendai 980, JAPAN

\begin{abstract}
Rayleigh-Fano theory has been extended for the purpose of calculating the polacization anomaly of a grating having hallow grooves and finite conductivity. simple analytic formulas are derived for predicting the position and the appearance of the anomalies. Phenomenological explanation are given to the origin of the anomaliea. The valic ity of our analysie is examined by comparing computed degree of polarization with experi zental data obtained in the vieible region for Al-, Ag-, and Au-coated blazed gratings.
\end{abstract}

\title{
1. INTRODUCTION
}

Grating anomalies and their ascociated problems have been a ubject of theorat.cal interest. The integral and the differential method are capable of solving guch probsems with the aid of a large computer'. However, they have come difficulties in obtairing physical ingight into the diffraction process involved. On the other hand, the surface plasmon theory ${ }^{2,3}$ gives a physical interpretation on the process, but it is lacking. in capability in explaining to a large extent the effect of the groove profile.

Despite the problems in its mathematical formalism, the classical Rayleigh-Fano theory is very attractive because it provides a clear physical insight into the diffraction processes involved and permita the use of a personal computer for computation. Therefore, we have extended the Rayleigh-Fano theory and applied it to the analysis of polarization anomalies of shallow metalic gratings.

We outline our extended Rayleigh-Fano theory' in Sec. 2. In Sec. 3, we derive Bimple analytic formulas for predicting the position and the appearance of anomalies and give phenomenological explanationg to the origin of the anomalies. We examine the validity of our analysis in Sec. 4 by comparing computed degree of polarization with the data measured in the visible region for $\mathrm{Al}-\mathrm{Ag}$ - and $\mathrm{Au}$ - coated blazed gratings.

\section{EXTENSION OF RAYLEIGH-FANO THEORY}

We define a Cartesian coordinate system and the positive direction of angles as shown in Fig. 1. The surface of a plane grating having a period $d$ in the $x$ direction and $z=$ $f(x)=f(x+d)$, divides the whole space into halves; the upper half, $U_{1,}$ is a vacuum and the lower half, $D_{2}$, is composed of the grating material having a complex refractive index $\bar{n}$. The monochromatic plane wave of wavelength $\lambda$ travels in a direction perpendicular to the grooves and impinges on the surface at an angle $\theta$. We approximate the fields on both sides of the gurface, that is, the incident field $u^{\text {til }}$, reflected diffraction field $v^{(r)}$, and refracted diffraction field $u^{(t)}$, by superposition of plane waves:

$$
\begin{aligned}
& u^{(i)}=\exp (i k(x \sin \theta-z \cos \theta)\} \text {, }
\end{aligned}
$$

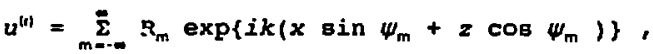

$$
\begin{aligned}
& u^{\prime \prime \prime}=\sum_{m=-.}^{\infty} D_{m} \exp \left\{i k n \tilde{n}\left(x \sin \phi_{m}-z \cos \phi_{m}\right)\right\} \text {, }
\end{aligned}
$$


where a time dependence of the form exp(-iwt) is implied, $\omega$ being the angular frequency of the incident wave. In Eq. (1), $k=2 \pi / \lambda, \psi_{\mathrm{m}}$ ig the diffraction angle of the reflected $m$ th order diffraction wave, $\phi_{m}$ is the diffraction angle of the refracted $m$ th order wave, and $R_{m}$ and $D_{m}$ are, respectively, the complex amplitudes of the reflected and refracted mth order diffraction waves.

We express the groove profile in a Fourier serieg

$$
z=f(x)=\sum_{p=-\infty}^{\infty}\left[a_{p} \cos (2 p \pi x / d)+b_{p} \sin (2 p \pi x / d)\right]=\sum_{p=1}^{\infty} f_{p} \exp (-i k l p x),
$$

where $f_{0}=0$ and $I=\lambda / d$, Using Eqa. (1) and (2), we expand raxwell's boundary conditions on the grating surface to the socond order in $f(x)$. Equating the coefficients of exp($i k l m x$ ) on both eides of the expanded boundary sonditione for a given $m$, we obtain

$$
\begin{aligned}
& G_{2 m}\left(\cos \psi_{m}+\beta \cos \phi_{m}\right) R_{m}=\left(G_{1 m}+H_{1 m}\right) \cos \theta-G_{1 m} \beta \cos \phi_{m}-i k 1 m f_{m} \ln \theta \\
& \left.-\sum_{m=m} R_{p}\left[\left(G^{\prime}{ }_{2 m e}+H^{\prime}{ }_{2 m p}\right) \cos \psi_{p}+G^{\prime}{ }_{2 m} \beta \cos \phi_{m}+i k\right](m-p) f_{m p} \sin \psi_{p}\right]
\end{aligned}
$$

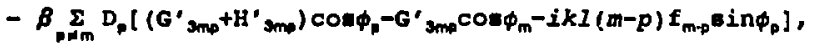

$$
\begin{aligned}
& G_{3 m}\left(\cos \psi_{m}+\beta \cos \phi_{m}\right) D_{m}=\left(G_{1 m}+H_{1 m}\right) \cos \theta+G_{1 m} \cos \psi_{m}-i k 1 m f_{m} \oplus i n \theta \\
& \text { - } \left.\sum_{m m} R_{p}\left[\left(G^{\prime}{ }_{2 m p}+H^{\prime}{ }_{2 m q}\right) \cos \psi_{p}-G^{\prime}{ }_{2 m p} \cos \psi_{m}+i k\right](m-p) f_{m-p} \operatorname{Bin} \psi_{p}\right] \\
& -\sum_{p=m} D_{p}\left[\beta\left(G^{\prime}{ }_{3 m p}+H^{\prime}{ }_{3 m p}\right) \cos \phi_{p}+G^{\prime}{ }_{3 m p} \cos \psi_{m}-i k I(m-p) f_{m-p} \beta B i n \phi_{p}\right] \text {, }
\end{aligned}
$$

where $\beta=\bar{n}$ for $s$ component, $\beta=1 / \bar{n}$ for $p$ component. The explicit expressions for $G_{\mathrm{m}}$ ' $G^{\prime}$ impe $H^{\circ}$ imp $\left(i=1,2,3\right.$ ) and $H_{1 m}$ in Eqs. (3) and (4) are

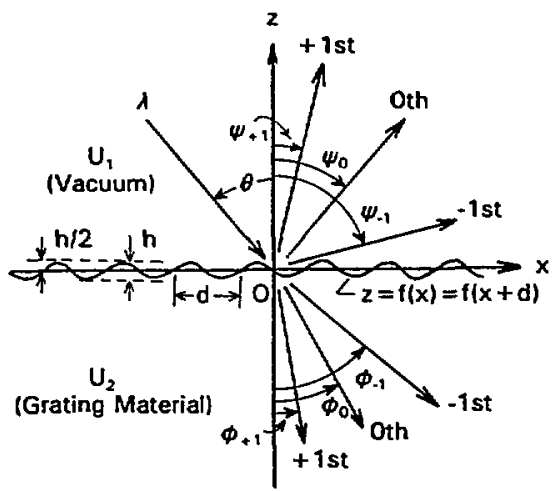

$$
\begin{aligned}
& G_{10}=1-\frac{1}{2} k^{2} g(0) \cos ^{2} \theta, \\
& G_{1 m}=-i k f_{m} \cos \theta-\frac{1}{2} k^{2} g(m) \cos ^{2} \theta,(m \neq 0), \\
& G_{2 m}=1-\frac{1}{2} k^{2} g(0) \cos ^{2} \psi_{m}, \\
& G_{2 m p}^{\prime}=i k f_{m p} \cos \psi_{p}-\frac{1}{2} k^{2} g(m-p) \cos ^{2} \psi_{p}, \\
& G_{3 m}=1-\frac{1}{2} h^{2} k^{2} g(0) \cos ^{2} \phi_{m}, \\
& G_{3 m p}^{\prime}=-i k n f_{m p} \cos \phi_{p}-\frac{1}{2} k^{2} n^{2} g(m-p) \cos ^{2} \phi_{p}, \\
& H_{1 m}=k^{2} I h(m) \sin \theta, \\
& H_{2 m p}^{\prime}=k^{2} I h(m-p) \sin \psi_{p}, \\
& H^{\prime}{ }_{3 m p}=k^{2} I n h(m-p) \sin \phi_{p},
\end{aligned}
$$

Eig. 1. Diffraction of a plane wave by a plane grating. The angles are taken as positive when measured in the direction indicated by arrows.

where

It is noted that both $R_{m}$ and $D_{m}$ are expresed as a linear combination of two kinds of terms. One is the functions of guantities associated only with the incident light ( $\lambda, \beta$ ), diffraction order $(m)$, groove profile $\left(d, f_{p}\right)$, grating material (ñ), and grating mounting $\left(\theta, \psi_{m}\right)$. The other is the terms that represent contributions from diffracted waves (both reflected and refracted) of the other orders. For a mirror, $i . e ., f_{p}=0$, the contributions from all the diffracted waves vanish, and Eqs. (3) and (4) are reduced to Fresnel formulas.

To deterinine the complex amplitudes $R_{m}$ and $D_{m}$ of the diffracted $m$ th order waves from the grating, we employ an iterative method with the initial values given by 


$$
\begin{aligned}
& \mathbf{R}_{m}{ }^{10)}=\frac{\left(G_{1 m}+H_{1 m}\right) \cos \theta-G_{1 m} \beta \cos \phi_{m}-i k I m f_{m} \sin \theta}{G_{2 m}\left(\cos \psi_{m}+\beta \cos \phi_{m}\right)}, \\
& D_{m}{ }^{101}=\frac{\left(G_{1 m}+H_{1 m}\right) \cos \theta+G_{1 m} \cos \psi_{m}-i k 1 m f_{m} \sin \theta}{G_{3 m}\left(\cos \psi_{m}+\beta \cos \phi_{m}\right)} .
\end{aligned}
$$

These initial values are the zero-order solutions obtained by neglecting the contributions from the diffracted $p(\neq m)$ th-order waves, 1.0. , by neglecting the terms containing $R_{p}$ and $D_{p}(p \neq m)$ in Eqs. (3) and (4). Iterations are carried out by succesaive approximation: (1) the lst order solutions $R_{m}^{\prime \prime \prime}$ and $D_{m}{ }^{\prime \prime \prime}$ are computed from Eqs. (3) and (4) by substituting the

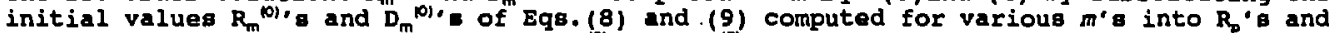
$D_{p} \cdot B,(2)$ the 2 nd order solutions $R_{m}^{(2)}$ and $D_{m}^{(2)}$ are computed from Eqs. (3) and (4) by substituting $R_{m}^{\prime \prime \prime} \cdot$ and $D_{m}^{\prime \prime \prime} \cdot g$ for $R_{p}^{\prime}$ and $D_{p}^{\prime}{ }^{\prime \prime}$, and $(3)$ higher-order solutions are obtained successively by repeating steps similar to (2). Iterations are terminated by referring to the degree of convergence of the solution and the energy balance criterion

$$
1=\underset{m \in U_{1}}{\sum} E_{m}+\underset{m \in U_{2}}{\sum E_{m}^{\prime}} \text {. }
$$

In Eq.(10), $E_{m}$ and $E_{m}^{\prime}$ are the energies of the reflected and tive refracted ath-order diffraction waves, respectively, and are expressed by

$$
E_{m}=R_{m} R_{m} \cdot \cos \psi_{m} / \cos \theta, E_{m}^{\prime}=\beta D_{m} D_{m}{ }^{\circ} \cos \phi_{m} / \cos \theta,
$$

where the asterisk means the complex conjugate.

To examine the validity of our method, we computed P-A curves, the degree of polarization $P$ va. wavelength $A$,

$$
P(m, \lambda)=\frac{\left[E_{m}(\lambda)\right]_{1}-\left[E_{m}(\lambda)\right]_{p}}{\left[E_{m}(\lambda)\right]_{1}+\left[E_{m}(\lambda)\right]_{p}},
$$

and the energy sums

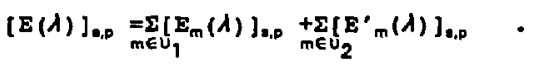

\section{ANALYSIS OF DIEERACTION ANOMALIES}

A physical picture of the grating anomaly can be drawn by examining the formula for the $n t h-o r d e r$ solution $R_{m}^{(n)}$. Applying successive approximations $n$ times, we obtain the complex amplitude $R_{\pi,}^{m /}$ of the reflected mth-order diffraction wave:

$$
R_{m}^{(n)}=R_{m}^{\text {NI }}+\sum_{p \circ m}^{\Sigma} \frac{F(\lambda, \theta, d, m, p, \beta)}{\left(\cos \psi_{m}+\beta \cos \phi_{m}\right)\left(\cos \psi_{p}+\beta \cos \phi_{p}\right)} f_{p} f_{m-p}+\text { higher orders, }
$$

where $F$ is a slowly varying function of $\lambda, \theta, \alpha, m, p$, and $\beta$ and is given by

$$
\begin{aligned}
F= & -k^{2}\left\{\left(\cos ^{2} \theta-\beta \cos \theta \cos \phi_{p}+1 p \sin \theta\right)\left[\cos ^{2} \psi_{p}+\beta \cos \psi_{p} \cos \phi_{m}+1(m-p) \sin \psi_{p}\right]\right. \\
& \left.-\beta\left(\cos ^{2} \theta+\cos \theta \cos \psi_{p}+1 p \sin \theta\right)\left[\bar{n} \cos ^{2} \phi_{p}-\bar{n} \cos \phi_{p} \cos \phi_{m}+1(m-p) \operatorname{gin} \phi_{p}\right]\right\} .
\end{aligned}
$$

The second term in Eq. (14) represents interactions among the diffracted waves of various orders $(\mathrm{p} \neq \mathrm{m})$ and the grating structure. These interactions may produce an anomalous effect on the mth-order spectrum under observation if the $p$ th-order wave is either diffracted again (by the grating) or reradiated resonantly (through momentum transfer with the grating) into the mth-order spectrum. This means that in order to have an anomaly, the pth-order wave must be nearly in the state of passing off $\left(\left|\sin \psi_{0}\right|=1\right)$ and the factor 
$\left(\cos \psi_{p}+\beta \cos \phi_{p}\right)$ must take a very small value rapidly as $\psi_{p}$ approachea $\pm 90^{\circ}$.

We observe the mth-order spectrum at a constant angle of incidence while increasing the wavelength $A$ of the incident light. The factor $\left(\cos \psi_{p}+\beta \cos \phi_{p}\right)$ is expressed as

$$
\begin{aligned}
& \cos \psi_{p}+\beta \cos \phi_{D}=\left\{\cos \psi_{D}+\operatorname{Re}\left(\beta \cos \phi_{p}\right)\right\}+i\left\{\operatorname{Im}\left(\beta \cos \phi_{p}\right)\right\} \text { for } \lambda \leq \lambda_{A}\left(\left|\sin \psi_{D}\right| \leq 1\right), \\
& \cos \psi_{p}+\beta \cos \phi_{p}=\left\{\operatorname{Re}\left(\beta \cos \phi_{p}\right)\right\}+i\left\{\left|\cos \psi_{p}\right|+\operatorname{Im}\left(\beta \cos \phi_{p}\right)\right\} \text { for } \lambda>\lambda_{A}\left(\left|\sin \psi_{D}\right|>1\right),
\end{aligned}
$$

where $\lambda_{n}$ is the Rayleigh wavelength of the pth-order wave and is defined by

$$
p \lambda_{n}=d(\sin \theta \pm 1) .
$$

Thus, an anomaly may occur when the real (imaginary) part of Eq. (16) (Eq. (17)) becomea zero at a certain $\lambda \leq \lambda_{n}\left(\lambda>\lambda_{n}\right)$.

For p- ( a-) polarized incident light, the real ( imaginary) parte of Ega. (16) and (17) are shown to be alwaye positive and are expected to be slowly varying function of $\lambda$. The imaginary part of Eq. (16) and the real part of Eq. (17) are also expected to vary slowly with $\lambda$. Therefore, no anomaly should occur, within the framework of a bhallow grating, at $\lambda \leq \lambda_{k}$ and $\lambda>\lambda_{n}$, respectively, for the $p$ - and the s-polarized light.

For the p-polarized light, an anomaly associated with the pth-order wave (in a trapped mode within the grating surface) is expected to occur at $\lambda>\lambda_{\mathrm{h}}$ that satisfies

$$
\left|\cos \psi_{p}\right|+\operatorname{Im}\left(\beta \cos \phi_{p}\right)=0 \text {, }
$$

if the following conditions are fulfilled:

$$
\begin{aligned}
& \left|\operatorname{Re}\left(\beta \cos \phi_{p}\right)\right| /\left|\operatorname{Im}\left(\beta \cos \phi_{p}\right)\right| \ll 1, \\
& \operatorname{Im}\left(\beta \cos \phi_{p}\right) \ll 0 \text { and }\left|d\left(\cos \psi_{p}\right) / d \lambda\right|>\left|d\left[\operatorname{Im}\left(\beta \cos \phi_{p}\right)\right\} / d \lambda\right| .
\end{aligned}
$$

For the g-polarized light, an anomaly associated with the pth-order wave (in a propagating mode) may occur at $A<\lambda_{k}$ that satisfies

$$
\cos \psi_{p}+\operatorname{Re}\left(\beta \cos \phi_{p}\right)=0 \text {. }
$$

if

$$
\begin{aligned}
& \left|\operatorname{Im}\left(\beta \cos \phi_{p}\right)\right|,\left|\operatorname{Re}\left(\beta \cos \phi_{p}\right)\right| \ll 1, \\
& \operatorname{Re}\left(\beta \cos \phi_{p}\right)<0 \text { and }\left|\mathrm{d}\left(\cos \psi_{p}\right) / \alpha \mu\right|>\left|\mathrm{d}\left[\operatorname{Re}\left(\beta \cos \phi_{p}\right)\right] / \alpha \lambda\right| .
\end{aligned}
$$

are satisfied. Equations (20) and (23) are the conditions of resonance with a small damping. The second relations in Eqs. (21) and (24) are the conditions necegsary for the anomaly to occur in a narrow wavelength range. In the visible range, highly reflective metals have extinction coefficients which are larger than the respective refractive indices. For this reason, Eq. (23) is violated, but Eq. (20) is not. Therefore, shallow metallic gratings do not exhibit anomalies for s-polarized light, but they may show anomalies at $\lambda>A_{R}$ for p-polarized light if $E q g .(20)$ and (21) are fulfilled.

\section{COMPARISON OF NUMERICAL RESULTS WITH EXPERIMENTAL DATA}

\subsection{General features of anomalieg}

To examine the validity of our analygis of the grating anomaly, we compare in this gection the numerical regults with some experimental data on polarization anomalies of Al-, Ag-, and Au-replica concave gratings having a radius of curvature of $0.5 \mathrm{~m}$, a groove density of 600 grooves/mm, and a blaze angle of $2^{\circ} 35^{\circ}$. Although the experimental data referred to here are on concave gratings, it was shown that the concavity of the grating surface has no influence on the anomalies if the aperture is kept smaller than $f / 13$, thus permitting us to use the data for comparison. 
Numerical computations were carried out by using a 80386-sX based personal computer and Basic corpiler. In the numerical computationg we used the values of optical constants for aluminum ${ }^{10.12}$, silver $^{13}$ and gold ${ }^{14}$ given in references. The groove profiles of the gratings were approximated by Fourier series of a finite length with $|p| \leq 10$. The degree of polarization was computed for 200 wavelengths in the measured wavelength range of $300-$ $700 \mathrm{~nm}$ at equal intervals. The energy sum was taken over apectral orderg from -10 to 10 for both the 8 and $p$ components. Under these conditions, it took 6 hours to compul:e twenty p- $\lambda$ curves $(m= \pm 1, \pm 2, \cdots, \pm 10)$ with five consecutive iterations and the respective energy sums.

Figure 2 showg the meagured (open circlea) and the computed (solld linea) P-A curves, the degree of polarization $P$ vs. wavelength $\lambda$, of $A 1-$, Ag-, and Au-coated gratings in lst order at a fixed incident angle of $5^{\circ}$. For reference, the Rayleigh wavelengths are indicated in Fig. 2 (a) by arrows and the aseociated paseing-off orders by numerals. All the $P-A$ curves shown here are obtained at the first iteration, which lo the best compromise as judged by the energy balance criterion. It is clearly seen in Fig. 2 (a) that the general features of the observed anomalies, 1.e., the position, shape, and appearance, are well reproduced by the computed curves. Slight diacrepancies between the observed and the computed $P-\lambda$ curves may be ttributed to oome extent to ambiguity in the values of blaze angle and optical constants usad for calculations. In the case of Alcoated grating, rather large deviattong seen in the dip positions at ghort wavelengths are most part due to $\mathrm{Al}_{2} \mathrm{O}_{3}$ layer formed on the grating surface.

\subsection{Position and sharpness of anomalies}

To curpare the computed regults with the observed data more in detail, we take up the P-A curves of Fig. 2 (a) as a typical example and consider the p-anomalies asgociated with

(a)
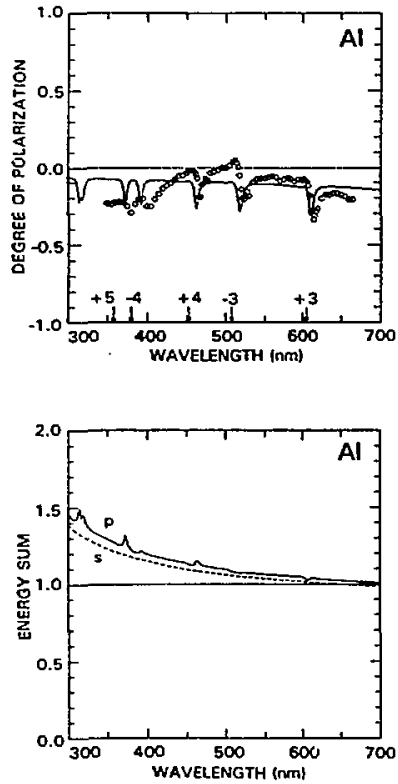
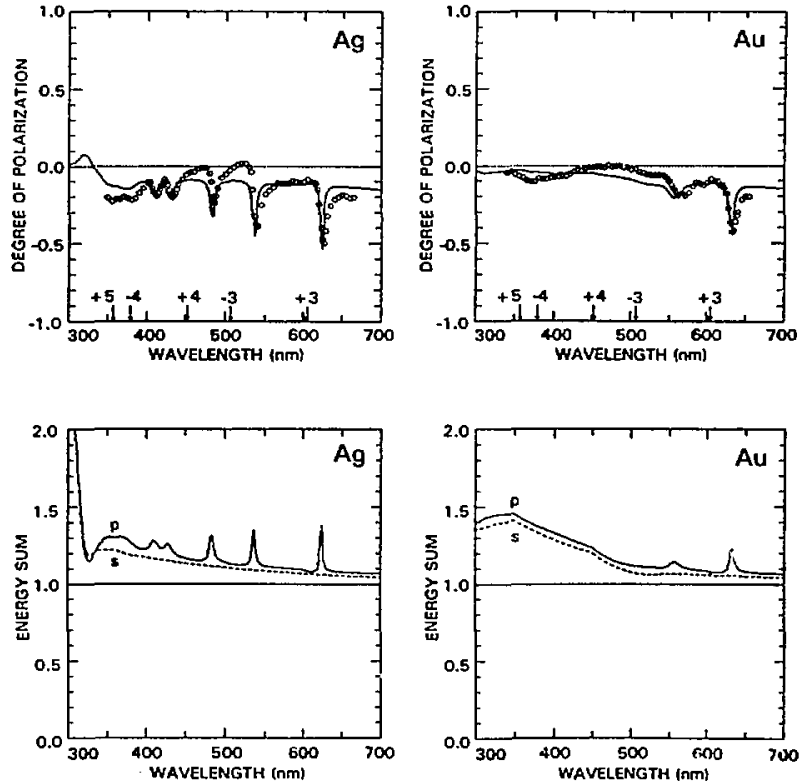

Fig. 2. Computed P- $\lambda$ curves (a) and the energy sums (b) obtained at the 1st iterations for 600-grooves $/ \mathrm{mm}, 2^{\circ} 35^{\prime}-\mathrm{blaze}, \mathrm{Al}-, \mathrm{Ag}-$ and Au-coated ruled concave gratings at a fixed angle of incidence of $5^{\circ}$. The corresponding experimental datampints(o) are shown in (a) for comparison. 
pasging-off orders of +3 and +4 . In this example, we can separate the effect of grating materials from that of groove profiles because the data of Fig. 2 (a) were taken with the gratings replicated in succession from one and the same replica grating using different grating materials.

Figure 3 shows for the Al-, Ag-, and Au-coated gratings the behavior of $-\left|\cos \psi_{\mathrm{p}}\right|$ and $\operatorname{Im}\left(\beta \operatorname{cosa} \phi_{p}\right)$ in the vicinity of the Rayleigh wavelength, $A_{n}=604.0 \mathrm{~nm}$ and $452.9 \mathrm{~nm}$ associated, respectively, with the passing-off order of +3 and +4 . As is geen in Fig. 3 , the two curves $-\left|\cos \psi_{p}\right|$ and $\operatorname{Im}\left(\beta \cos \phi_{p}\right)$ intersect at a wavelength $\lambda_{0}$. This implies the fulfillment of Eq. (19), suggenting a posibility of obgerving an anomaly at $\lambda_{0}\left(A_{0}{ }^{\prime}\right.$ are found to be $611.2,623.1$, and $631.1 \mathrm{~nm}$, in Fig. 3 (a) and $462.8,482.9$, and $503.3 \mathrm{~nm}$ in Fig. 3 (b), respectively, for the Al-, Ag-, and Au-conted gratings.

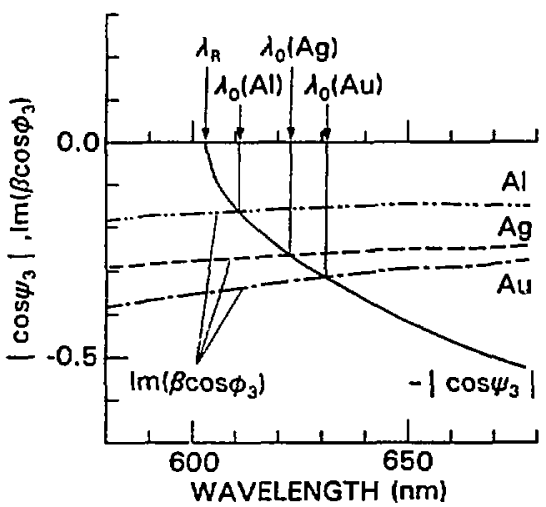

(a)

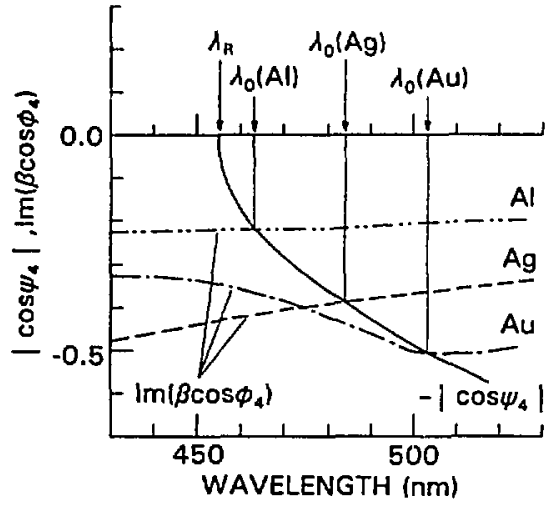

(b)

Fig. 3. $-\left|\cos \psi_{p}\right|$ and $\operatorname{Im}\left(\beta \cos a \phi_{p}\right)$ vs. wavelength in the vicinity of anomalies associated with (a) $p=+3$ and (b) $p=+4$ for the $A l-$, $A g-$, and $A u-c o a t e d ~ g r a t i n g s$.

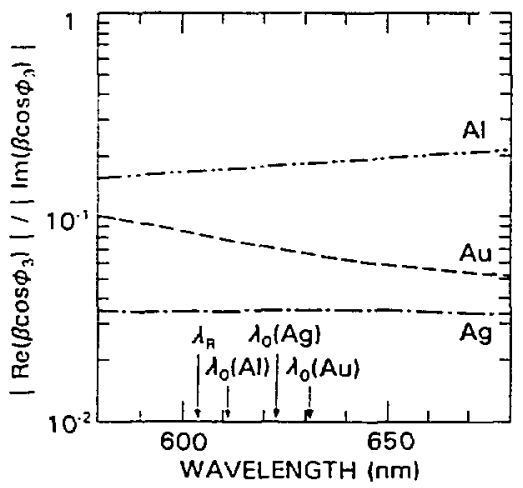

(a)

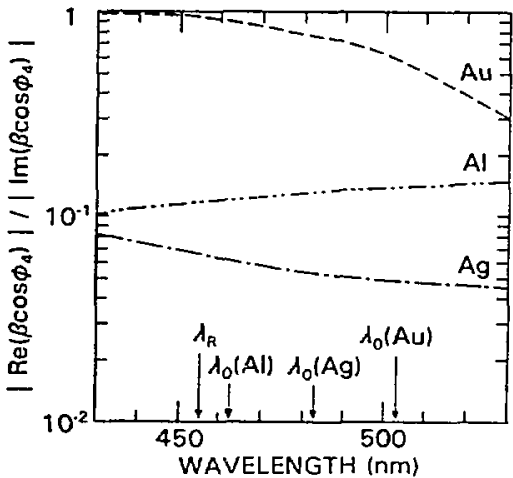

(b)

Fig. 4. The magnitude of of $\left|\operatorname{Re}\left(\beta \cos \phi_{p}\right)\right| /\left|\operatorname{Im}\left(\beta \cos \phi_{p}\right)\right|$ vs. wavelength in the vicinity of anomalies associated with (a) $p=+3$ and (b) $p=+4$ for the AI-, Ag- and Au-coated gratings. 
The damping effect due to the coating materials (cf. Eq. (20)) should be examined in order to judge whether the anomalies under consideration really appear or not. To do this, we plot $\left\{\operatorname{Re}\left(\beta \cos \phi_{p}\right)|/| \operatorname{Im}\left(\beta \cos \phi_{p}\right) \mid\right.$ against wavelength as shown in $F i g$. 4 . As to the anomalies agsociated with $p=+3$, the damping effect are fairly small for all the gratings (see Fig. 4 (a)), suggesting occurrence of anomalieg. The atrength of anomalies are estimated, from Fig. 4 (a), to be in order of the Ag-, Au-, and Al-coated gratings. ThiB estimation agrees with the observed result. In the case of $p=+4$ (Fig. 4 (b)), the Agand Al-coated gratings show fairly gmall damping, whereas the Au-coated grating has strong damping. Therefore, in the cage of the Au-coated grating, no anomaly could be excited by the diffracted wave with the passing-off order of +4 . This expectation is in agreement with the observed results.

\section{CONCLUSION}

An attempt has been made to analyze the diffraction anomalies of a grating having shallow grooves and finite conductivity by meane of an extended Rayleigh-Fano theory. We derived imple formulas for predicting the wavelength position and the sharpnegs of anomalies. Condidering the theoretical and experimental results obtained, it is concluded that the basic features of the diffraction anomalies can be easily predicted by our method. The theory also provides explanations about the effect of the groove profile on the appearance of anomalies, and thie subject will be treated elsewhere.

\section{ACKNOWLEDGMENT}

This work was partly upported by Material Scienceg Division of the U.S. Department of Energy under contract No. DE-AC03-76SF00098.

\section{REFERENCES}

1. R. Petit, Ed., Electromagnetic Theory of Gratings, Topics in current Physics Vol. 22 (Springer-Verlag, Berlin, 1980).

2. R. H. Ritchie, E. T. Arakawa, J. J. Cowan and R. N. Hanm, "Surface-Plasmon Resonance Effect in Grating Diffraction," Phys. Rev. Letters 21, 1530-1533 (1968).

3. C. E. Wheeler, E. T. Arakawa and R. H. Ritchie, "Photon Excitation of Surface Plasmons in Diffraction Gratings: Effect of groove Depth and Spacing," Phys. Rev. B13, 2372-2376 (1976).

4. Lord Rayleigh, "On the Dynamical Theory of Gratings," Proc. Roy. Soc. (London) $\mathbf{A 9}$, 399-416 (1907); gee also Theory of Sound (Dover Pub., New York, 1945), 2nd ed., Vol.II, Chapt. 13, Sec. 272a,pp. 89-96.

5. U. Fano, "Zur Theorie der Intensitätsanomalien der Beugung," Ann. Phys. 32, 393-443 (1938).

6. U. Fano, "The Theory of Anomalous Diffraction Gratings and of Quasi-stationary Waves on Metallic Surfaces (Sommerfeld's Waves)," J. Opt. Soc. Am. 31, 213-222 (1941).

7. M. Koike and T. Namioka, "An Extended Rayleigh-Fano Theory and Its Application to Grating Polarization," submitted to Appl. Opt.

8. K. Saito and T. Namioka, "Polarization Anomalieg of Concave Gratings," Jpn. J. Appl. Phys. 19, 607-613 (1980).

9.K. Saito, H. Noda and T. Namioka, "Polarization Charactoristics of Concave Gratings Hounted in a Seya-Namioka Honochromator," Opt. Acta 25, 1055-1071 (1978).

10. G. Hass and J. E. Waylonis, "Optical Constants and Reflectance and Transmittance of Evaporated Aluminum in the Visible and Ultravlolet," J. Opt. Soc. Am. 51, 719-722 (1961).

11. L. G. Schulz, "The Optical Constantis of Silver, Gold, Copper, and Aluminum. I. The Absorption Coefficient $k, " J$. Opt. Soc. P.m. 44, 357-361 (1954).

12.L. G. Suhulz and F. R. Tangherlini, "Optical constants of silver, Gold, Copper, and Aluminum. II.The Index of Refraction, "J. Opt. Soc. Am. 44, 362-368 (1954).

13.D. W. Lynch and W. R. Hunter, "Comments on the optical Constants of Metals and Introduction to the data for Several Metis $18, "$ in Handbook of optical Constants of Solids, E. D. Palik ed., (Academic Press, Orlando, 1985), pp.275-367.

14.N. Bashara and D. Peterson, "Ellipsometer Study of Anomalous Absorption in Very Thin Dielectric Films on Evaporated Metals," J. Opt. Soc. Am. 36, 1320-1331 (1946). 